\title{
Correlation between Acoustic Emissions Generated from Cavitation in Rubber Tree Leaf Veins
}

\author{
Kensuke Kageyama, Mitsuru Kaminaga and Hiroshi Kato \\ Graduate School of Science and Engineering, Saitama University, Saitama 338-8570, Japan
}

(Received December 25, 2006)

\begin{abstract}
Cavitation causes embolisms followed by a considerable decrease in xylem conductance: embolisms pose a serious obstacle to the continued uptake of water. Assuming that acoustic emissions (AEs) with high similarity would be generated from cavitation at the same element when refilling occurred, this study investigated the generation of such an $\mathrm{AE}$ in a rubber tree's leaf vein. Cross-correlation analyses between the filtered AE waveforms showed the existence of AEs with a cross-correlation coefficient greater than 0.97. That result indicated that some AEs came from the same element in a leaf vein where cavitation and refilling occurred repeatedly. Assuming that a pair of AEs with a high cross-correlation coefficient corresponded to the same embolism, the relationship between daily diameter change (DDC) and number of embolisms showed a better linear relation than that between DDC and total events of AE.
\end{abstract}

Keywords : cavitation, cross-correlation analysis, embolism, strain gauge, transpiration

\section{INTRODUCTION}

A sudden phenomenon, called cavitation, is known to occur in xylem conduits or leaf veins of plants when water moves through the soil-plant-atmosphere continuum under negative pressure (Milburn, 1966). Cavitation causes embolisms followed by a considerable decrease in xylem conductance: embolisms pose a serious obstacle to the continued uptake of water (Tyree and Sperry, 1989). Ultrasonic techniques have been applied to the study of cavitation since the 1970s. Those results show that the measured acoustic emissions (AE) are associated with cavitation (Milburn, 1973). Although many plants have been examined to measure AE caused by water stress, the rate of $\mathrm{AE}$ occurrence in many plants occasionally deviates from the transpiration rate (Bormann et al., 2001). Therefore, it is still difficult for acoustic emission methods to diagnose the water status of plants. A possible reason explaining why the rate of AE occurrence does not reflect the transpiration rate is xylem refilling after cavitation events. Recent studies have shown that embolized vessels are repaired even when the water in neighboring vessels is under tension (Tyree et al., 1999; Holbrook et al., 2001). Cavitation will occur at the same element of the vessel repeatedly if refilling occurs after cavitation followed by the generation of high negative stress. In such cases, the cumulative number of AEs will not correspond to the total number of embolized elements. However, a strong similarity pertains between the AEs generated from cavitations at the same element when no change occurs in microstructure and water distribution along the AE's propagation path. The number of the classified AE groups will be related to the total number of embolized ele-

Corresponding author: Kensuke Kageyama, fax : +81-48-856-2577, e-mail : kageyama@mech.saitama-u.ac.jp 


\section{K. KAGEYAMA ET AL.}

ments. For that reason, the evaluation of hydraulic conductivity of vessels will be possible if the $\mathrm{AE}$ waveforms can be classified with high statistical cross-correlation. The rate of AE occurrence should be kept low to preserve the conditions under which no change occurs in the propagation path of the AEs. The change in microstructure or water distribution in a stem will not be negligible if the rate of plant growth is too high or if cavitation events are too numerous. Eventually, the similarity between the AEs generated from the same cavitated element would be lost.

The first purpose of this study is to investigate whether the AEs generated from xylem conduits have high similarities. A rubber-tree leaf vein was selected as a material to measure AEs. Rubber trees have high resistance against water stress and high hydraulic conductance. Furthermore, the amount of water transferred through a leaf vein is much less than that through a trunk. It is expected that the rate of AE occurrence at a leaf vein is low. The second aim is to compare the number of classified AE groups with the negative pressure in the stem. A strain gauge has been used in previous studies for measurement of the diameter change of stems or trunks (Link et al., 1998). Moriya et al. showed that the stem diameter changed deeply depending on the negative pressure in the stem (Moriya et al., 1992). To measure diameter variations of tree trunks, a strain gauge was used because it offers many advantages over other methods: it is small and lightweight, with a low price and high reliability. For those reasons, the strain gauge was used in this study to measure the negative pressure in a leaf-vein.

\section{MATERIALS AND METHODS}

A potted dwarf miniature rubber tree (Ficus elastica) was cultivated using a hydroponic system with water and a fired clay medium during $12 \mathrm{~h}$ light exposure each day. Figure 1 shows that the $30-\mathrm{cm}$ tall rubber tree was transferred into a Faraday cage with artificial light irradiated from a fluorescent lamp. The temperature was set to $30^{\circ} \mathrm{C}$ and the pot was put in a tray of water. Light intensities at the top and base of the shoot were 60 and $20 \mu \mathrm{mol} \mathrm{m}^{-2} \mathrm{~s}^{-1}$, respectively and the lighting cycle ran $12 \mathrm{~h}$ of light per day. The experiments were done using high $\left(40^{-} 60 \mu \mathrm{mol} \mathrm{m}^{-2} \mathrm{~s}^{-1}\right)$, medium $\left(27-40 \mu \mathrm{mol} \mathrm{m}^{-2} \mathrm{~s}^{-1}\right)$, and low $\left(20-27 \mu \mathrm{mol} \mathrm{m}^{-2} \mathrm{~s}^{-1}\right)$ irradiation levels. A leaf was clamped with an AE sensor; a strain-gauge sensor was used for each experiment, as shown in Fig. 2, to measure $\mathrm{AE}$ and the diameter change of a leaf vein simultaneously.

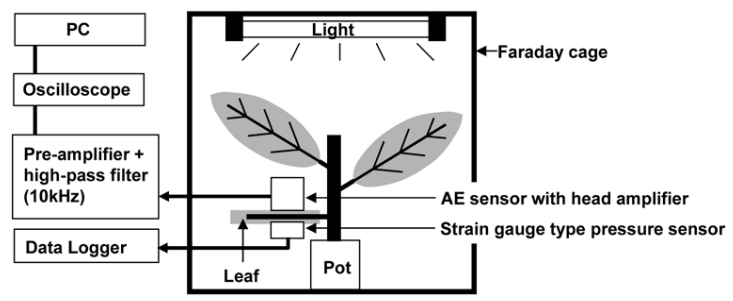

Fig. 1 Schematic and photograph of the growth chamber and AE measuring system.

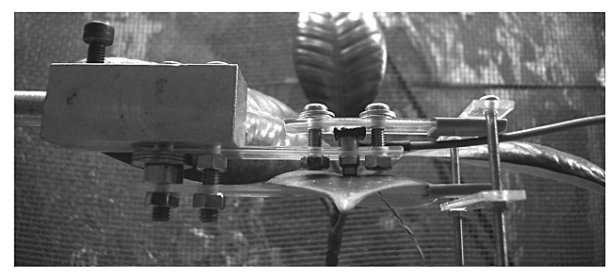

Fig. 2 Setup for clamping a leaf vein with an AE sensor and a strain gauge sensor. 


\section{ACOUSTIC EMISSIONS IN RUBBER TREE}

A piezoelectric AE sensor with a head amplifier (M304A; Fuji Ceramics Corp.) was put on the upper side of a leaf vein. The AE signal was amplified using the head amplifier and a preamplifier with a high pass filter set at $10 \mathrm{kHz}$ to filter out low-frequency sounds. The total gain of this system was $69 \mathrm{~dB}$; only $\mathrm{AE}$ data above $150 \mathrm{mV}$ at output were considered for analyses. Because measured AE waveforms were transferred into a personal computer via GP-IB, the dead time at $\mathrm{AE}$ acquisition was $600 \mathrm{~ms}$. That time is longer than that of commercial AE measuring systems, but it is acceptable for this experiment because total AE counts per day were less than a hundred.

A strain-gauge type pressure sensor (PDA-PA-200KPA; Tokyo Sokki Kenkyujo Co., Ltd.) was used as a displacement gauge. The sensor diameter is $6.5 \mathrm{~mm}$; a 4-mm-diameter rubber pad was attached to a diaphragm. Calibration against the displacement was done with compression test using a micrometer. The calibration result is shown in Fig. 3. A first-order linear approximation was applied at strains of -600 to $-200 \mu \varepsilon$, within which the measured values of all experiments were obtained. The strain-gauge sensor was put on the lower side of a leaf vein that was clamped with acrylic bars under a compressive load that was weaker than $-1.29 \mathrm{~N}(-600 \mu \varepsilon)$.

The AE and strain measurement at each experiment started a few minutes after clamping a leaf vein. The strain was recorded for three days every $5 \mathrm{~min}$ using a data logger. For transpiration measurements, the pot was put on a strain-gauge type weighing scale and the actual loss of weight was recorded daily. The examined rubber tree received water only at the beginning of each experiment. The pot was bagged in a plastic bag to prevent evaporation. The daily transpiration rate (g/day) was calculated by dividing the daily weight loss by the total area of leaves of the examined rubber tree.

\section{RESULT AND DISCUSSION}

Diameter change and AE occurrence behavior

Figure 4 shows typical diameter change and AE occurrence behavior. The daily average leaf

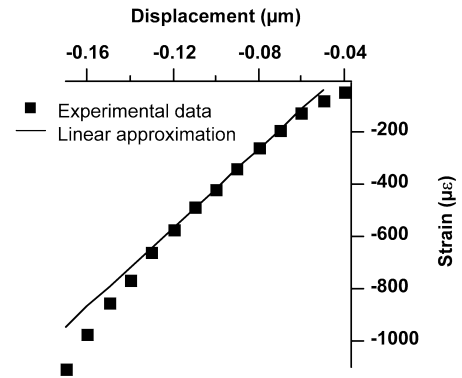

Fig. 3 Strain sensor calibration against displacement.

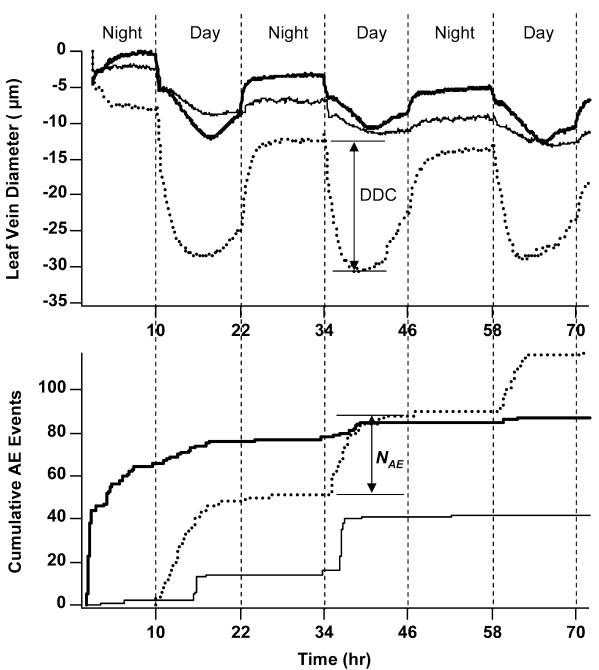

Fig. 4 Typical diameter changes and AE occurrence behavior. Solid and bold lines: medium irradiated (samples M1 and M4, respectively). Dotted line: low irradiated (sample L3). 


\section{K. KAGEYAMA ET AL.}

vein diameter was gradually degraded by stress relaxation. A sudden increase in leaf-vein pressure resulted in stress relaxation, which is commonly observed in organic materials when a leaf vein is clamped by a pair of sensors. Stress relaxation is related to the viscoelastic properties of a leaf vein: it is irrelevant to water status or transpiration. During daytime, the leaf-vein diameter decreased, reached a minimum value, and then increased again. This behavior coincides with the results obtained in other studies, which reported that daily variation in stem diameter is caused by the negative pressure in the conduits (Ueda and Shibata, 2001). Therefore, the diameter change of a leaf vein during daytime is inferred to correspond to the negative pressure in the leaf vein. Figure 4 also indicates that less irradiated leaves showed higher diameter change during daytime. The increased leaf temperature causes an absolute concentration difference of water vapor between the leaf air space and the bulk air outside the leaf, which makes the driving force for water loss from the leaf (Taiz and Zeiger, 2002). More-irradiated leaves might decrease their leaf aperture to prevent excessive water loss. The diameter change, the negative pressure, in the leaf vein was eventually decreased, corresponding to the irradiation in the experiments in this study.

The AE occurrence behavior during daytime indicated that cavitation occurred with the leafvein shrinkage because most $\mathrm{AE}$ were measured at the time that the leaf vein diameter was decreasing. Moreover, AEs were measured occasionally during the first night from the beginning of measurement. That AE occurrence is caused by compressive deformation that is induced when the leaf vein is clamped. Such a deformation decreases the water conductance, thereby engendering cavitation because of the partial shortage of water.

Figure 5 (A) shows the relationship between the whole transpiration rate and the daily diameter change (DDC). Figure 4 shows that the DDC was defined as the difference between maximum and minimum diameter during daytime. The DDC varied widely among leaves and showed no correlation with the overall transpiration rate: It indicated that the water status of each leaf would widely vary between leaves even if the overall transpiration rate and irradiation were constant. Figure 5 (B) shows the total number of AE events during daytime on each day $\left(N_{\mathrm{AE}}\right)$; the same behavior as that of DDC is apparent for the overall transpiration rate. The $N_{\mathrm{AE}}$ was loosely related to

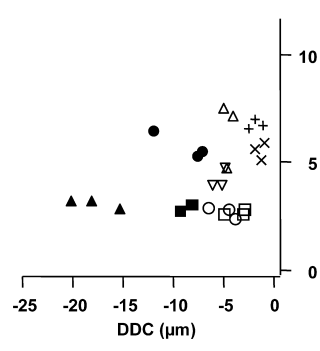

(A)

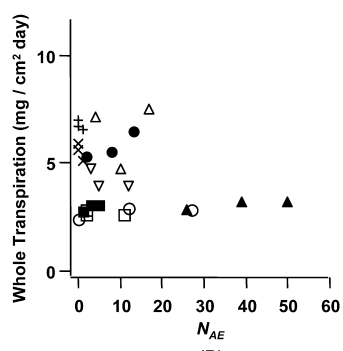

(B)

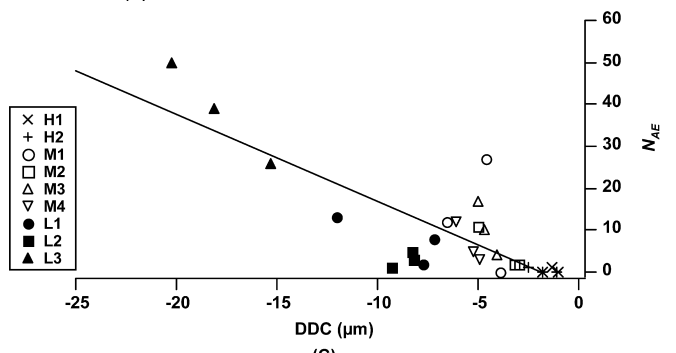

(C)

Fig. 5 Relationship between daily diameter change (DDC), AE events and whole transpiration. $\mathrm{H} 1$ and $\mathrm{H} 2$ were high irradiated, M1, M2, M3 and M4 were medium irradiated, and L1, L2 and L3 were low irradiated. 


\section{ACOUSTIC EMISSIONS IN RUBBER TREE}

DDC, as shown in Fig. 5 (C): higher negative pressure engendered more cavitation. Figure 5 (C), however, indicated that the value of $N_{\mathrm{AE}}$ of some samples, e.g., M1, deviated from the least-squares line of all data and thus suggested that cavitation was occasionally generated regardless of the diameter change of leaf veins, or the negative pressure in leaf veins.

Cross-correlation analysis of AE waveforms

Figure 6 shows typical AE waveforms measured from sample M1 in the morning at the second day. The waveforms of Fig. 6 are apparently classifiable into two types: (B, C, E, F, G and I) and (A, D, H and J). Cross-correlation analyses were carried out to evaluate the similarity of the waveforms. First, the response property of AE sensor should be considered because the crosscorrelation coefficient tends to rise when the peak frequency of a waveform is near the resonance frequency of the sensor.

Figure 7 shows the average frequency spectrum of the background noises measured using AE sensor a thousand times randomly; it shows that the AE sensor used in this study has a resonance frequency of $510 \mathrm{kHz}$. To extract the $\mathrm{AE}$ waveforms measured in the frequency domain where the response frequency spectrum of the AE sensor was smooth, a ninth-order band-pass filter (100-400 $\mathrm{kHz}$ ) was processed using software that simulated the characteristics of an RC filter.

Then, cross-correlation analysis was carried out between the filtered AE waveforms within 20 $\mu$ s around the maximum peak of each waveform. Table 1 shows the results of the cross-correlation

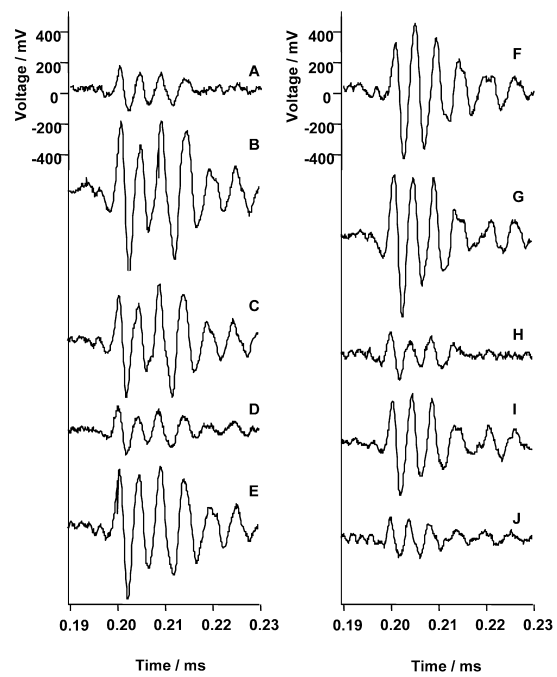

Fig. 6 Typical AE waveforms measured from sample M1 on the morning of the second day $(9: 24-9: 29)$.

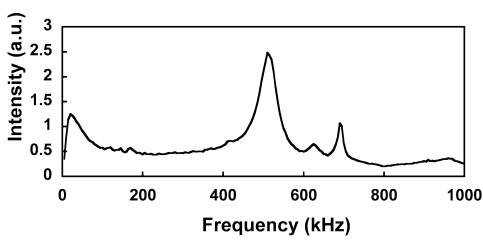

Fig. 7 Average frequency spectrum of the background noises measured using an AE sensor.

Table 1 Cross-correlation coefficients of the waveforms shown in Fig. 6.

\begin{tabular}{cccccccccc}
\hline & $\mathrm{A}$ & $\mathrm{B}$ & $\mathrm{C}$ & $\mathrm{D}$ & $\mathrm{E}$ & $\mathrm{F}$ & $\mathrm{G}$ & $\mathrm{H}$ & $\mathrm{I}$ \\
\hline $\mathrm{B}$ & 0.827 & & & & & & & & \\
$\mathrm{C}$ & 0.862 & 0.995 & & & & & & & \\
$\mathrm{D}$ & 0.981 & 0.911 & 0.935 & & & & & & \\
$\mathrm{E}$ & 0.809 & 0.993 & 0.989 & 0.889 & & & & & \\
F & 0.848 & 0.978 & 0.984 & 0.909 & 0.991 & & & & \\
G & 0.818 & 0.998 & 0.992 & 0.899 & 0.997 & 0.984 & & & \\
H & 0.991 & 0.859 & 0.887 & 0.993 & 0.830 & 0.856 & 0.843 & & \\
I & 0.870 & 0.987 & 0.995 & 0.932 & 0.991 & 0.996 & 0.990 & 0.883 & \\
J & 0.989 & 0.835 & 0.874 & 0.979 & 0.811 & 0.847 & 0.823 & 0.986 & 0.875 \\
\hline
\end{tabular}




\section{K. KAGEYAMA ET AL.}

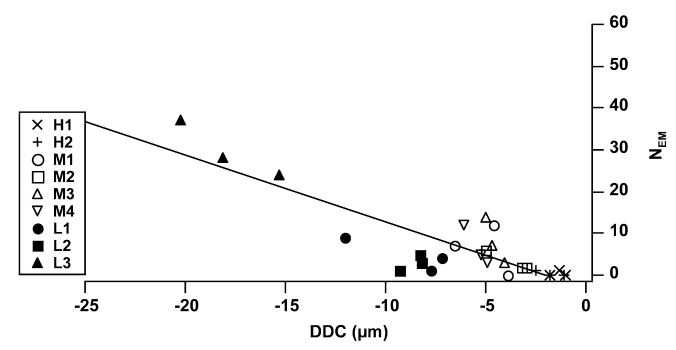

Fig. 8 Relationship between daily diameter change (DDC) and number of embolized elements.

analysis between waveforms in Fig. 6: the waveforms were classified into black or gray groups where pairs of waveforms had a cross-correlation coefficient greater than 0.97. Assuming that the AEs with the high cross-correlation coefficient correspond to the embolism at the same element, the AEs shown in Fig. 6 came from the two sites in the leaf vein at which cavitation and refilling occurred repeatedly: these AEs showed that only two leaf-vein elements were embolized.

Using the procedure mentioned above, cross-correlation analyses were carried out between the AEs measured during daytime on each day. When a pair of AEs with a cross-correlation coefficient greater than 0.95 was found, both AEs of the pair were classified into a same AE group. Then, the number of embolisms of each day were derived assuming that the AEs of a same group corresponded to a same embolism, i.e., if newly measured AE had a cross-correlation efficient greater than 0.95 with the $\mathrm{AE}$ that already occurred on the day, the newly measured $\mathrm{AE}$ would be assumed to be no count for embolism. Figure 8 shows the relationship between DDC and the number of embolized elements $\left(N_{\mathrm{EM}}\right)$ and the number of classified AE groups. The linear relation is improved. Therefore, AE classification using cross-correlation analysis seems to be reasonable to evaluate the number of embolized elements. The AE from a same element should have the constant waveform to apply this technique to other plants. Plants with high transpiration rates, however, often showed much higher AE rates than the rubber tree in this study: over a hundred per hour. In such a case, the change in water conditions in a stem or leaf vein cannot be negligible. For example, if another embolism occurs in the propagation path of an $\mathrm{AE}$, the waveform of the measured $\mathrm{AE}$ would be different from that without embolism. Consequently, the AE classification using cross-coefficient analysis is a useful method to evaluate embolism conditions of plants with low rate of cavitation, but further study should be applied to plants with high rate of cavitation.

Authors thank Fuji Ceramics Corp. for additional technical assistance on AE sensors.

\section{REFERENCES}

Bormann, M. W., Ernst, M. K., Liebig, H. P. 2001. Concurrent non-destructive measurement of ultrasonic acoustic emission and transpiration on broccoli. J. Appl. Bot. 75: 173-177.

Holbrook, N. M., Ahrens, E. T., Burns, M. J., Zwieniecki, M. A. 2001. In vivo observation of cavitation and embolism repair using magnetic resonance imaging. Plant Physiol. 126: 27-31.

Link, S., Thiede, M., Babel, M. V. 1998. An improved strain-gauge device for continuous field measurement of stem and fruit diameter. J. Exp. Bot. 49: 1583-1587.

Milburn, J. A. 1966. The conduction of sap. I. Water conduction and cavitation in water stressed leaves. Planta 65: 34-42.

Milburn, J. A. 1973. Cavitation studies on whole Ricinus plants by acoustic detection. Planta 112: $333-342$.

Moriya, H., Iwao, K., Kageyama, H. 1992. Studies on non-destructive and continuous measurement of water contents and applications to irrigation in crop culture. Acta Hortic. 304: 345-351. 


\section{ACOUSTIC EMISSIONS IN RUBBER TREE}

Taiz, L., Zeiger, E. 2002. Plant physiology. Third Edition. ch. 4, web topic 4.5, Sunderland, Sinauer Associates.

Tyree, M. T., Sperry, J. S. 1989. Vulnerability of xylem to cavitation and embolism. Annu. Rev. Plant Physiol. Plant Mol. Biol. 40: 19-38.

Tyree, M. T., Salleo, S., Nardini, A., Gullo, M. A., Mosca, R. 1999. Refilling of embolized vessels in young stems of laurel. Do we need a new paradigm? Plant Physiol. 120: 11-21.

Ueda, M., Shibata, E. 2001. Diurnal changes in branch diameter as indicator of water status of Hinoki Cypress. Trees 15: 315-318. 